Preprint typeset in JHEP style - HYPER VERSION

McGill-04/12

\title{
Dimensionless Coupling of Bulk Scalars at the LHC
}

\author{
P.-H. Beauchemin, ${ }^{1,2}$ G. Azuelos ${ }^{1,3}$ and C.P. Burgess ${ }^{2}$ \\ ${ }^{1}$ Laboratoire René J.-A. Lévesque, Université de Montréal, \\ C.P. 6128, Montréal, Québec, Canada, H3C 3J\%. \\ 2 Physics Department, McGill University, 3600 University Street, \\ Montréal, Québec, Canada, H3A $2 T 8$ \\ 3 TRIUMF, 4004 Wesbrook Mall, Vancouver BC, V6T $2 A 3$
}

\begin{abstract}
We identify the lowest-dimension interaction which is possible between Standard Model brane fields and bulk scalars in 6 dimensions. The lowest-dimension interaction is unique and involves a trilinear coupling between the Standard Model Higgs and the bulk scalar. Because this interaction has a dimensionless coupling, it depends only logarithmically on ultraviolet mass scales and heavy physics need not decouple from it. We compute its influence on Higgs physics at ATLAS and identify how large a coupling can be detected at the LHC. Besides providing a potentially interesting signal in Higgs searches, such couplings provide a major observational constraint on 6D large-extra-dimensional models with scalars in the bulk.
\end{abstract}




\section{Contents}

1. Introduction 1

2. Lowest-Dimension Bulk Scalar Couplings 3

3. $h-\phi$ Production at Colliders 5

4. More Detailed Simulations 8

4.1 Standard Model Backgrounds 9

4.2 Analysis 9

5. Conclusions 14

6. Acknowledgments 16

\section{Introduction}

One of the most surprising observations of recent years has been the recognition that extra dimensions could be larger than a micron across [1] without being inconsistent with observations. This picture is consistent with the relative strength of gravity and the weak interactions provided that three properties hold: $(i)$ there are only two dimensions which are this large (for a total of 6 , including the 4 dimensions we see); (ii) the scale of gravity in the extra dimensions is of order a TeV; and (iii) ordinary particles (apart from the graviton) are trapped on a 3-brane, and so are unable to probe the existence of the large dimensions.

Not surprisingly, this observation has spurred considerable phenomenological study of the signatures for large extra dimensions at machines like the Large Hadron

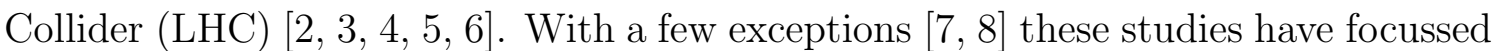
exclusively on the implications of gravitational physics in the bulk, partly because this physics is the least model-dependent. ${ }^{1}$ In broad terms these studies have concluded that bulk gravitational physics can be detectable at accelerators like the LHC if the extra-dimensional gravity scale, $M_{g}$, is of order a few TeV. On the other hand,

\footnotetext{
${ }^{1}$ Under the rubric 'gravitational' we include also discussions of 4D scalars and vectors which arise as components of the higher-dimensional metric. The situation for large extra dimensions is in sharp contrast with studies of extra dimensions which are much smaller than micron scales, for which a variety of phenomenological studies of potential LHC signals have been investigated.
} 
astrophysical bounds 9, 10 typically require lower bounds like $M_{g} \gtrsim 10 \mathrm{TeV}$, making it not clear whether observational effects should be expected at colliders.

Recently there have been several new proposals which suggest a re-examination of the implications of these kinds of large-extra-dimensional (LED) models for experiments, starting from several different motivations. In particular, large extra dimensions for which the bulk is supersymmetric are being studied because of their potential implications for the cosmological constant [11, 12] as well as because of the novel, non-MSSM realization of weak-scale supersymmetry breaking which they imply 13.

These models should predict a very rich phenomenology at the TeV scale, which is only now beginning to be explored. Among the new features which they imply is the existence of a multitude of new states living in the bulk which are necessarily light and weakly coupled because they are related to the graviton by supersymmetry, and because supersymmetry is only broken in the bulk at energies smaller than 1 eV. Furthermore, these models have plausible ultraviolet completions (like string theory) which imply numerous new states at $\mathrm{TeV}$ energies, such as massive string modes, Kaluza Klein or winding modes associated with additional TeV-scale extra dimensions. Indeed the existence of these new states (many of which are expected to be much lighter than $M_{g}$ ) may allow the astrophysical bounds on $M_{g}$ to be reconciled with the existence of observable effects at colliders [13].

In this paper we explore a different way in which LED models might produce observable effects at the LHC, despite having the gravity scale at $10 \mathrm{TeV}$ or higher. This new mechanism relies on the existence on our brane of the ordinary Higgs doublet (as would be the case if the brane physics includes the Standard Model), and on the existence of a fundamental scalar in the 6D bulk which is a singlet under any bulk gauge transformations, and is not an exact Goldstone boson (and so does not have a shift symmetry, of the form $\phi \rightarrow \phi+\epsilon+\ldots)$.

If these two kinds of scalars exist, then gauge invariance and general covariance allow them to couple to the brane scalars through a trilinear interaction whose effective coupling, $a$, is dimensionless and so is unsuppressed by inverse powers of the extra-dimensional gravity scale, $M_{g}$. Because we have ruled out symmetries which forbid such a coupling, it is likely to be generated by loops even if it is excluded at the classical level. We shall see that this makes the coupling plausibly of a size which could be observed at the LHC, regardless of the size of $M_{g}$.

We calculate the sensitivity to such a coupling at the LHC, and find that searches for the Higgs using its decay into 2 photons can probe effective couplings down to $a \gtrsim 0.09$. They can do so because of the new process, $p p \rightarrow h \phi \rightarrow \gamma \gamma \mathbb{E}_{T}$, which this new effective interaction opens up. ${ }^{2}$ The signal for higgs production in association

\footnotetext{
${ }^{2}$ The signal of Higgs production in association with missing energy to which we are led resembles that of the 4-dimensional model ref. [14], which was proposed as the most minimal model for dark matter.
} 
with bulk scalars turns out to be fairly easy to detect because none of the Standard Model backgrounds involve appreciable missing energy.

Our presentation is organized as follows. The next section describes this lowestdimension interaction, and summarizes its properties. This is followed, in section 3 , by a calculation of the parton-level process of $h \phi$ production through gluon fusion, as well as the ancillary calculations which are required in order to promote the parton process into a cross section for the hadronic reaction $p p \rightarrow h \phi$. Section 4 then presents the results of detailed simulations of this process, modelling the signals which would be seen within the ATLAS detector. In particular, we describe in this section how the significance of the signal depends on the various cuts which can be imposed. Our conclusions are briefly summarized in Section 5.

\section{Lowest-Dimension Bulk Scalar Couplings}

In the class of models of present interest, all Standard Model particles reside on a 3brane embedded within a 6 -dimensional bulk space, whose gravity scale is $M_{g} \sim 10$ $\mathrm{TeV}$. Indeed, it is conservative to assume that the particle content on our brane consists of no other fields besides those required by the Standard Model [13.

In general the bulk can be populated by a variety of degrees of freedom, but within the context of supersymmetric large extra dimensions (SLED) the bulk particle content would consist of the states of one of the varieties of 6-dimensional supergravity. For instance, the fields of 6D supergravity might consist of [15, 16] the metric, $g_{M N}$, 2-form gauge potential, $B_{M N}$, a dilaton (scalar), $\phi$, plus their fermionic partners: the gravitino, $\psi_{M}$, and dilatino, $\chi$. In addition there may also be $6 \mathrm{D}$ matter multiplets, such as might be required by anomaly-cancellation for a chiral supergravity [17]. These could involve gauge multiplets (containing a gauge potential, $A_{M}$, and gaugino, $\lambda$ ), hyper multiplets (involving scalars, $\varphi^{i}$, and fermions, $\omega^{a}$ ) or others.

Although the bulk couplings of these fields are dictated by supersymmetry, the absence of supersymmetry on the branes permits the bulk-brane couplings to be more complicated. In general, whatever these bulk-brane couplings are, the most important ones for phenomenological purposes are usually those having the lowest dimension, since these typically dominate the effective field theory at energies, $E \ll$ $M_{g} \cdot{ }^{3}$ For this reason we focus our attention in this paper on the lowest-dimension coupling which is allowed between the generic bulk fields and the (purely StandardModel) fields on our brane.

For any model with bulk $6 \mathrm{D}$ scalars there is a unique interaction which is possible between a bulk scalar and a brane-bound Standard Model field for which the effective

\footnotetext{
${ }^{3}$ We follow here the spirit of ref. [18, which gives a similar discussion of the phenomenology of the lowest-dimension couplings of Standard Model fields in 4 dimensions.
} 
coupling involves a non-negative power of mass. This is the effective trilinear coupling between a general bulk scalar $\phi(x, y)$ (not necessarily the dilaton), and the Standard Model Higgs doublet, $H(x)$ :

$$
S_{\text {int }}=-a \int d^{4} x \sqrt{-g} H^{\dagger} H(x) \phi\left(x, y_{b}\right),
$$

where $a$ is the dimensionless coupling. We use here coordinates $x^{\mu}, \mu=0,1,2,3$ to label dimensions parallel to the Standard Model brane, and $y^{m}, m=4,5$, for those dimensions transverse to the brane. $y^{m}=y_{b}^{m}$ denotes the position of the Standard Model brane within the bulk. All other effective interactions necessarily have couplings which are suppressed by inverse powers of $M_{g}$, assuming only that the bulk fields are singlets under the Standard Model gauge group.

In principle, it is possible for an interaction like eq. (2.1) to be forbidden by symmetries of the bulk, such as by shift symmetries $\delta \phi=\epsilon+\ldots$ which would occur if $\phi$ were a Goldstone boson. This kind of symmetry is quite common in higherdimensional supergravities, for which the bulk scalars are often the coordinates of coset spaces, $G / H$. Such a coupling can appear for the $6 \mathrm{D}$ dilaton, depending on the microscopic origin of the dilaton and of the 3-brane in question. ${ }^{4}$

It must be borne in mind, though, that even should the coupling of eq. (2.1) not be present classically, unless precluded by symmetries it will be generated by loops and the size of the resulting contribution to $a$ can be significant given the non-decoupling of high mass scales and the strengths of the bounds we shall find below. A representative size for such a coupling as generated by one loop in 6 dimensions might then be set by loop-counting factors, like $a \sim N /(4 \pi)^{3}$ with $N$ denoting the number of particle species circulating in the loop. For the simplest $6 \mathrm{D}$ supergravities [15] $N$ is typically $O(10-20)$, while for chiral $6 \mathrm{D}$ supergravities the requirements of anomaly cancellation can imply $N \gtrsim O(1000)$. Depending on the number of fields which contribute, an estimate for the loop-induced coupling could well be $a \sim O(0.01-1)$. We shall see that couplings at the upper end of this range might be observable at the LHC.

In unitary gauge, we have $H=\left(\begin{array}{c}0 \\ v+h(x)\end{array}\right)$ where $v$ is the expectation value which breaks the electroweak gauge group, and in the absence of bulk-brane couplings $h(x)$ is the physical scalar field. In terms of this, the coupling to the bulk field becomes

$$
S_{i n t}=-a \int d^{4} x \sqrt{-g}(v+h)^{2} \phi\left(x, y_{b}\right),
$$

\footnotetext{
${ }^{4}$ For instance, if the $6 \mathrm{D}$ dilaton is essentially the $10 \mathrm{D}$ dilaton, then a coupling like eq. (2.1) is present if the 3-brane arises as a D5 or D7 brane wrapped about a 2- or 4- cycle in the dimensional reduction to 6 dimensions from 10. It does not arise classically if the 3-brane is a D3 brane or is an NS5-brane wrapped on a 2-cycle. In the mechanism of cosmological constant suppression in the 6D SLED proposal there is no dilaton-brane coupling at the classical level [12], because of the classical scale invariance which this mechanism presupposes.
} 
We see that three separate kinds of couplings are implied between the bulk scalar, $\phi$, and the Higgs scalar, $h$ : $(a)$ a linear potential for $\phi$ of the form $a v^{2} \phi ;(b)$ a bulkbrane mixing term of the form $a v h \phi$; and $(c)$ a trilinear coupling of the form $a h^{2} \phi$. We ignore the first of these since it acts to shift the ground state of $\phi$ away from the value determined by the bulk scalar potential, and none of our results depend on this value in any case.

The second type of interaction implies a mixing between $h$ and the various Kaluza-Klein modes of the bulk scalar, $\phi$. This mixing is removed by diagonalizing the resulting scalar mass matrix, which for small $a$ leads to an $O(a)$ overlap between the physical Higgs state and the bulk KK modes. The physics of this mixing is very similar to previous discussions of gravi-scalar mixing with brane modes, and leads to a non-negligible invisible width [2, 4] for the Higgs particle. We do not further explore this width in detail, although its implications would be important to understand once the Higgs is discovered and its properties are being explored in detail.

In this paper we focus on the trilinear $h^{2} \phi$ interaction, which can play a role in the discovery signal of the Higgs. In the next sections we compute the implications of this coupling for Higgs production in association with missing energy.

\section{3. $h-\phi$ Production at Colliders}

The effective action

$$
S_{\text {int }}=-a \int d^{4} x \sqrt{-g} h^{2} \phi\left(x, y_{b}\right),
$$

can be used to compute the cross-section at tree-level for production of bulk scalars, radiated by a Higgs boson in $p-p$ collisions at the LHC. Since Higgs production at the LHC is dominated by gluon fusion, our interest at the parton level is therefore in the reaction $g g \rightarrow h \phi$, where both final-state particles are on shell. This process is similar to those studied in ref. [8], although the effective couplings of $\phi$ to fermions and gluons used in that reference have higher dimension, and so are likely to be dominated by the coupling to the Higgs used here (in models for which it is present).

For most values of the Higgs mass its dominant decays are hadronic, but such decay channels can be easy to miss because of the strong QCD background at the LHC. Consequently, we choose instead to study the more rare, but cleaner, $h \rightarrow$ $\gamma \gamma$ channel. The physical signal which bulk-scalar emission would produce in this channel is then two photons plus missing energy, as the scalar $\phi$ escapes into the extra dimensions.

We compute the Feynman rule for the $h-h-\phi$ vertex and derive the parton-level cross-section for producing a Higgs plus a bulk scalar in the final state. We follow here

the discussion of ref. [8], wherein the sum over the finely-spaced Kaluza-Klein states 


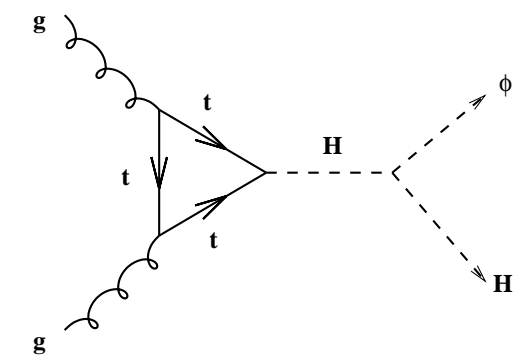

Figure 1: Parton-level Feynman graph which dominates Higgs/bulk-scalar production at the LHC.

of the $\phi$ field is replaced by the equivalent integral over the extra-dimensional phasespace of the bulk-scalar momentum. This, specialized to 2 large extra dimensions, gives the following phase-space measure (after integration over the angular variable):

$$
\int_{\Omega_{2}} \frac{d^{2} L}{(2 \pi)^{2}}=\frac{d M_{\phi}^{2}}{4 \pi} .
$$

Here we denote the 6 components of the bulk-scalar momentum by $\left\{\ell^{\mu}, L^{m}\right\}$, where $\ell^{\mu}$ denotes the components of motion parallel to the brane and $L^{m}$ denotes its components in the extra transverse dimensions. If $\phi$ has 6 -dimensional mass $\mu_{\phi}$ then $M_{\phi}^{2}=\mu_{\phi}^{2}+L^{m} L_{m}=-\ell^{\mu} \ell_{\mu}$ is the effective 4-dimensional mass the bulk scalar carries as seen by brane-bound observers due to its motion in the extra dimensions. In later applications we take $\mu_{\phi}=0$ because our interest lies with those scalars whose masses are kept light, such as they would be if they were related to a massless particle (like the graviton) by supersymmetry. Generalization of our results to arbitrary $\mu_{\phi}$ is straightforward.

The dominant amplitude at the parton-level is obtained by evaluating the Feynman graph of Fig. (1). ${ }^{5}$ In evaluating this graph we use the full momentum-dependent expression for the effective Higgs-gluon vertex which is obtained by evaluating the fermion sub-loop, along the lines of refs. [19]. It suffices to keep only the contribution of the top-quark loop, in which case the fermion loop leads to the following form factor for the effective Higgs-gluon coupling (for on-shell gluons)

$$
V_{g g h}=\frac{\alpha_{s}}{12 \pi v} \mathcal{F}\left(\frac{m_{t}^{2}}{Q^{2}}\right)
$$

where $Q^{2}=-q^{2}$, for $q^{\mu}$ the 4-momentum carried by the virtual Higgs. We follow the conventions of ref. [20], for which $\mathcal{F}(r)=3[2 r+r(4 r-1) f(r)]$ and

$$
f(r)=\left\{\begin{array}{cl}
-2\left[\arcsin \left(\frac{1}{2 \sqrt{r}}\right)\right]^{2} & \text { if } \quad r>\frac{1}{4} ; \\
\frac{1}{2}\left[\ln \left(\frac{\eta_{+}}{\eta_{-}}\right)\right]^{2}-\frac{\pi^{2}}{2}+i \pi \ln \left(\frac{\eta_{+}}{\eta_{-}}\right) & \text {if } \quad r<\frac{1}{4}
\end{array}\right.
$$

\footnotetext{
${ }^{5}$ Other graphs for which the bulk scalar is emitted by other particles give contributions which are suppressed relative to Fig. (11) by powers of external momenta divided by $M_{g}$.
} 
with $\eta_{ \pm}=\frac{1}{2} \pm \sqrt{\frac{1}{4}-r}$.

The form factor $\mathcal{F}(r)$ defined by the above expressions has the following wellknown properties [19]. It vanishes for small $r$, ensuring the negligible contributions of all low-mass quarks in the loop. For large $r$ it behaves as $\mathcal{F}(r)=1+O(1 / r)$. For intervening values of $r, \mathcal{F}(r)$ grows to a maximum for $r=O(1)$, falling off on either side towards the two asymptotic limiting forms. ${ }^{6}$

The differential parton-level cross-section obtained in this way is given by:

$$
\frac{d \sigma}{d \hat{t} d M_{\phi}^{2}}(g g \rightarrow h \phi)=\left(\frac{a^{2} \alpha_{s}^{2}}{144 v^{2}}\right) \frac{|\mathcal{F}|^{2}}{\left(\hat{s}-m_{h}^{2}\right)^{2}} .
$$

where $\mathcal{F}=\mathcal{F}\left(m_{t}^{2} / \hat{s}\right), m_{h}$ is the Higgs mass and $M_{\phi}$ is the effective bulk-scalar mass as defined above. The quantities $\hat{s}$ and $\hat{t}$ denote the usual parton-level Mandelstam kinematic variables. As usual, the integrated cross section grows like $M_{\phi}^{2}$, and so is dominated by the highest bulk-scalar masses, reflecting the enormous extradimensional phase space which is available for such states.

The cross-section for proton-proton collisions is obtained from this parton-level result in the usual way, by convoluting with the parton distribution functions, $f_{i}\left(x, Q^{2}\right)$. Since we are interested in the production of a real Higgs that decays into two photons for our later analysis, when performing these steps we compute the cross-section for the missing-energy process $p p \rightarrow \phi+h \rightarrow \gamma \gamma \mathbb{E}_{T}$. The result takes the following form:

$$
\sigma(p p \rightarrow h \phi)=\int d x_{1} d x_{2} d \hat{t} d M_{\phi}^{2}\left[f_{g}\left(x_{1}, Q^{2}\right) f_{g}\left(x_{2}, Q^{2}\right)\left(\frac{d \sigma(g g \rightarrow h \phi)}{d \hat{t} d M_{\phi}^{2}}\right)_{\hat{s}}\right]
$$

where $\hat{s}$ is related to the $p p$ centre-of-mass energy, $E_{c m}$, by $\hat{s}=x_{1} x_{2} E_{c m}^{2}$. Finally, we obtain the cross section for $p p \rightarrow \gamma \gamma \mathbb{E}_{T}$ by multiplying the above expression by the appropriate branching ratio, $B=B(h \rightarrow \gamma \gamma)$.

We have calculated this cross section and implemented the process in the generator PYTHIA [21]. We generate events randomly in phase space and assign weights to them. The events are then accepted or rejected proportionately to these weights by PYTHIA, which also performs the relevant hadronizations.

When performing the phase-space integrations we use the following constraints:

- We require the transverse momentum of the final Higgs particle to satisfy $P_{T}^{2}>$ $P_{c u t}^{2}$, where $P_{\text {cut }}$ is a minimum value which can be chosen at generation level. (We set $P_{\text {cut }}=0$ in the analysis below.) This implies that the variable $\hat{t}$ must lie in the range $t_{-} \leq \hat{t} \leq t_{+}$, with

$$
t_{ \pm}=\frac{1}{2}\left[\left(m_{h}^{2}+M_{\phi}^{2}-\hat{s}\right) \pm \sqrt{\left(m_{h}^{2}+M_{\phi}^{2}-\hat{s}\right)^{2}-4\left(m_{h}^{2} M_{\phi}^{2}+P_{c u t}^{2} \hat{s}\right)}\right] .
$$

\footnotetext{
${ }^{6}$ Because of the small rise in $\mathcal{F}(r)$ for $m_{t}^{2}<Q^{2}<4 m_{t}^{2}$, we find that use of the asymptotic expression $\mathcal{F} \approx 1$ underestimates the size of the cross section by roughly $20 \%$.
} 
- Energy-momentum conservation implies the following upper limit for $M_{\phi}^{2}$

$$
0 \leq M_{\phi}^{2} \leq M_{\text {max }}^{2}=\hat{s}+m_{h}^{2}-2 \sqrt{\hat{s}\left(m_{h}^{2}+P_{c u t}^{2}\right)} .
$$

- The parton energy fractions, $x_{i}$, lie in the range $x_{\min } \leq x \leq 1$, with:

$$
x_{m i n}=\frac{\hat{s}_{m i n}}{s}=\frac{P_{c u t}^{2}+\sqrt{P_{c u t}^{2}+m_{h}^{2}}}{s},
$$

and, as usual, $s$ denotes the Mandelstam initial-energy invariant for the full proton-proton collision.

\begin{tabular}{|c|c|c|c|c|c|c|c|c|}
\hline Higgs mass $(\mathrm{GeV})$ & 80 & 90 & 100 & 110 & 120 & 130 & 140 & 150 \\
\hline \hline Cross-section $(\mathrm{pb})$ & 34.2 & 27.4 & 22.5 & 18.8 & 15.9 & 13.6 & 11.8 & 10.3 \\
\hline Branching ratio $(\%)$ & 0.086 & 0.119 & 0.148 & 0.190 & 0.220 & 0.222 & 0.193 & 0.138 \\
\hline$\sigma \times B(\mathrm{fb})$ & 29.4 & 32.6 & 35.6 & 34.9 & 30.2 & 22.7 & 14.2 & 5.9 \\
\hline SM $p p \rightarrow h(\mathrm{pb})$ & 46.5 & 38.0 & 31.8 & 26.7 & 23.0 & 20.0 & 17.4 & 15.8 \\
\hline Mass resolution $(\mathrm{GeV})$ & 1.11 & 1.20 & 1.31 & 1.37 & 1.43 & 1.55 & 1.66 & 1.74 \\
\hline
\end{tabular}

Table 1: Cross-sections for the signal process $p p \rightarrow h \phi$ using the coupling value $a=0.5$, and for $p p \rightarrow h X$ as a function of the Higgs mass. Also shown are the branching ratio for Higgs decay into two photons and the mass resolutions $\left(\sigma_{H}\right)$ at high luminosity in ATLAS.

Table (四) shows the various pieces of the total cross section for $p p \rightarrow h \phi \rightarrow \gamma \gamma \mathbb{E}_{T}$ as a function of Higgs mass in the range $80 \mathrm{GeV}-150 \mathrm{GeV}$. The first row of the table gives the total cross-section for $p p \rightarrow h \phi$ (in $\mathrm{pb}$ ); the second row gives the branching ratio for the decay channel $h \rightarrow \gamma \gamma$ (in percent), and the third row multiplies these to give the cross section for $p p \rightarrow h \phi \rightarrow \gamma \gamma \phi$ (in fb). For comparison, the last two rows give the cross section for the Standard Model process $p p \rightarrow h X$, as well as the Higgs mass resolution in the ATLAS detector, for the process $h \rightarrow \gamma \gamma$, for high luminosity (as computed by the ATLAS collaboration in ref. [22]). For this table, the bulk-scalar process effective coupling constant, $a$, was set to 0.5 , so as to yield a $p p \rightarrow h \phi$ production cross section which is comparable to the SM process $p p \rightarrow h X$. This gives a rough indication of what size effective couplings might be observable, and so motivates the more detailed calculations which we now describe.

\section{More Detailed Simulations}

We now describe a more detailed analysis of the expected signals, including Standard Model backgrounds in a more systematic way, as well as incorporating detector effects. To do this we assign parton flavors in each event according to the 
CTEQ 5L parton distribution functions [23] evaluated at the renormalization scale $Q^{2}=\frac{1}{2}\left(m_{h}^{2}+M_{\phi}^{2}\right)+p_{T}^{2}$ and colour flow between these partons is applied. ATLAS detector effects were incorporated using the fast Monte Carlo program ATLFAST [24.

\subsection{Standard Model Backgrounds}

Since the bulk scalar, $\phi$, radiated by the Higgs would quickly escape into the extra dimensions, it should escape detection. The observed process is therefore $p p \rightarrow \gamma \gamma$ with missing energy in the final state, and so the backgrounds are the usual ones for the process $p p \rightarrow h \rightarrow \gamma \gamma$. As discussed in [22] these come in two types. First, there is an irreducible background consisting of genuine photon pairs produced by the Born process $(q \bar{q} \rightarrow \gamma \gamma)$, by the box diagram process $(g g \rightarrow \gamma \gamma)$ and by quark bremsstrahlung ( $q g \rightarrow q \gamma \rightarrow q \gamma \gamma$ ). Second there is also the reducible background consisting of QCD jet-jet or $\gamma$-jet events - in which one or both jets are misidentified as photons 22]. These two sources of background are comparable in size, even though the reducible backgrounds have huge cross-sections compared to the irreducible ones. This is because there are compensating large rejection factors thanks to the efficient photon/jet discrimination which is expected for ATLAS. These rejection factors have been evaluated to be $2 \times 10^{7}$ or $8 \times 10^{3}$ respectively, for jet-jet and $\gamma$-jet backgrounds. Once this rejection efficiency is included, the reducible background events number about $20 \%$ of the expected number of irreducible background events. To the above backgrounds, we add processes with much lower cross sections, but which include neutrinos in the final state. In particular, we consider the associated production processes $Z h \rightarrow \nu \bar{\nu} \gamma \gamma, W h \rightarrow \ell \nu \gamma \gamma$ and $t \bar{t} h, h \rightarrow \gamma \gamma$. We also take into account the processes $Z \gamma \gamma, Z \rightarrow \nu \bar{\nu}$ and $W \gamma \gamma, W \rightarrow \ell \nu$ which can also mimic the signal. All the backgrounds were generated with PYTHIA. For the cases of $Z \gamma \gamma$ and $W \gamma \gamma$, we simulated the processes $Z \gamma$ and $W \gamma$, with the second $\gamma$ arising from initial or final state radiation. A $p_{T}$ cut of $35 \mathrm{GeV}$ was applied in these cases.

Table (2) shows the cross-sections and the total number of events expected after the application of the above-mentioned rejection factors, for each background process. The table assumes an integrated luminosity of $100 \mathrm{fb}^{-1}$. Notice that a further reduction factor of around $80 \%$ must also be applied in addition due to the expected reconstruction efficiency of photons. Finally, following ref. [22], we incorporate the quark bremsstrahlung process into the simulation by scaling the two other irreducible backgrounds by $50 \%$ of the combined Born plus box contribution, after having applied isolation cuts in ATLFAST.

\subsection{Analysis}

We now describe a set of cuts which can be used to isolate those $p p \rightarrow \gamma \gamma$ events which also involve significant amounts of missing energy. We then use these cuts to quantify the smallest size for $a$ which can be expected to be detectable. 


\begin{tabular}{|c|c|c|}
\hline Processes & cross-section $(\mathrm{pb})$ & Number of events \\
\hline \hline$p p \rightarrow \gamma \gamma($ Born$)$ & 56.2 & $5.62 \times 10^{6}$ \\
\hline$p p \rightarrow \gamma \gamma(\mathrm{box})$ & 49.0 & $4.90 \times 10^{6}$ \\
\hline$p p \rightarrow$ jet+jet & $4.9 \times 10^{8}$ & $2.50 \times 10^{6}$ \\
\hline$p p \rightarrow$ jet $+\gamma$ & $1.2 \times 10^{5}$ & $1.50 \times 10^{6}$ \\
\hline$p p \rightarrow h \rightarrow \gamma \gamma$ & $4.63 \times 10^{-2}$ & 4630 \\
\hline$p p \rightarrow Z h, W h, t \bar{t} h$ & & 250 \\
$Z \rightarrow \nu \bar{\nu}, W \rightarrow \ell \nu, h \rightarrow \gamma \gamma$ & $2.5 \times 10^{-3}$ & $3.3 \times 10^{5}$ \\
\hline$p p \rightarrow Z \gamma ; Z \rightarrow \nu \bar{\nu}$ & 3.3 & $5.6 \times 10^{5}$ \\
\hline$p p \rightarrow W \gamma ; W \rightarrow \ell \nu$ & 5.6 &
\end{tabular}

Table 2: SM backgrounds to the production of bulk scalars in association with the Higgs at ATLAS, their cross-section (for an $E_{T}^{c u t}$ of $23 \mathrm{GeV}$ ) and the total number of events expected at ATLAS for an integrated luminosity of $100 \mathrm{fb}^{-1}$ (after application of rejection factors).

We first establish our criteria for identifying two isolated photons. In the ATLAS detector photons are detected if they are emitted with pseudorapidity in the range $|\eta|<2.5$. We consider such photons to be isolated if their transverse momentum satisfies $P_{T}^{\gamma}>5.0 \mathrm{GeV}$, and if there is less than $10 \mathrm{GeV}$ of energy deposited by all other particles within a cone of radius $\Delta R=\sqrt{(\Delta \phi)^{2}+(\Delta \eta)^{2}}<0.4$ around the photon of interest.

Part of the reducible background consists of jets which are misidentified as photons, and so we need also define our criteria for jet reconstruction. For this we use the cone algorithm, with a cone radius of $\Delta R=0.4$, a pseudorapidity coverage of 5.0 and a minimal jet energy threshold of $10 \mathrm{GeV}$.

The first cuts to be imposed are those which optimize the significance of the $h \rightarrow \gamma \gamma$ signal for the standard Higgs search at ATLAS [22]. To this end we require:

Cut 1: The two photon candidates, ordered in $p_{T}$, must have transverse momenta which are in excess of 40 and $25 \mathrm{GeV}$. (That is, we choose $P_{T}=40 \mathrm{GeV}$ for photon 1 and $P_{T}=25 \mathrm{GeV}$ for photon 2.)

Cut 2: Both photon candidates must lie in the pseudorapidity interval $|\eta|<2.4$ and have a pseudorapidity separation of at least $0.15(\Delta \eta>$ $0.15)$;

Cut 3: The reconstructed mass of the two photons, the two jets, or the jet $+\gamma$ final state must have an invariant mass which is sufficiently close to the Higgs mass. Quantitatively, we demand: $M_{H}-1.4 \sigma_{H}<M_{\gamma \gamma}<$ $M_{H}+1.4 \sigma_{H}$, where $\sigma_{H}$ is the $h \rightarrow \gamma \gamma$ resolution quoted in table (1). 

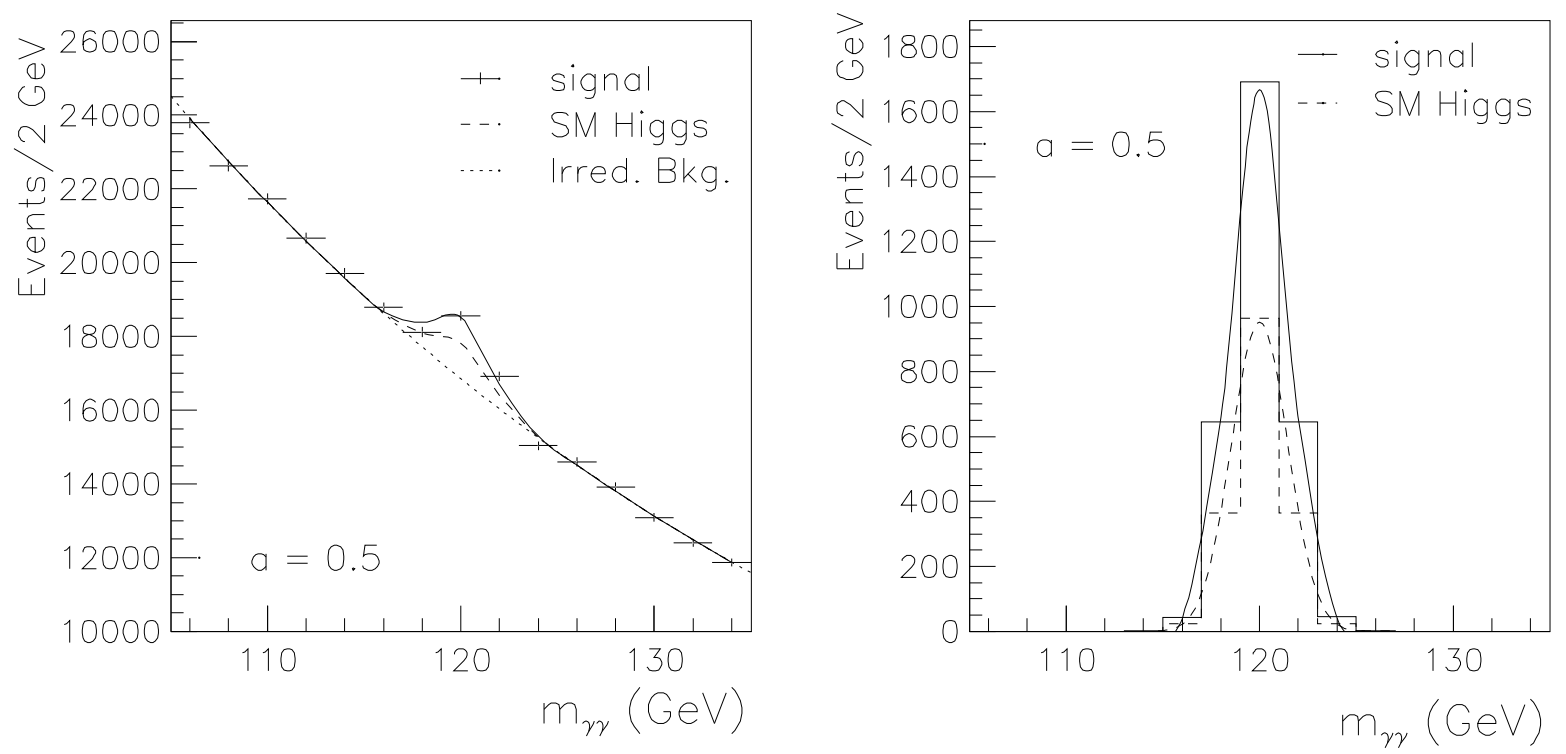

Figure 2: Expected $h+\phi$ signal for $M_{H}=120$. GeV, for an integrated luminosity of 100 $\mathrm{fb}^{-1}$ and with $h h \phi$ coupling value chosen as $a=0.5$. The left panel shows the signal on top of the irreducible background, while the right one shows the reconstruction of the mass peak.

Assuming an integrated luminosity of $100 \mathrm{fb}^{-1}$, after imposing these cuts and a $80 \%$ efficiency for detecting each photon, we are left with a total of 45,000 background events and 1,500 standard $h \rightarrow \gamma \gamma$ events. This leaves us with $38 \%$ of the initial number of signal events - $p p \rightarrow \gamma \gamma+\mathbb{E}_{T}$ — independently of $a$ (8930 events before the cut for $a=0.5)$.

In order to decide whether the missing-energy signal can be winnowed out of the background, we first recall that the previous ATLAS analyses [22] indicate that these same cuts would permit the standard Higgs signal to be identified with a significance of $6.2 \sigma$ (using $S / \sqrt{B}$ as the significance criterion). After cuts, an effective coupling of size $a=0.5$ produces roughly the same number of $p p \rightarrow h \phi$ events as from the Standard Model $p p \rightarrow h$ process, for $m_{h}=120 \mathrm{GeV}$, even if the cross section is somewhat lower. This is due to the fact that the final-state Higgs are more transverse in energy, leading to a larger acceptance of photons. Therefore, we roughly expect couplings of this size to be detectable at the $6 \sigma$ level given $100 \mathrm{fb}^{-1}$ of data. The significance for the Higgs signal itself is thus doubled. Since the $h \phi$ production rate scales like $a^{2}$, a coupling $a=0.44$ would correspond to a $5 \sigma$ significance. For couplings this large about half of the Higgs particles are produced in association with $\phi$ emission into the extra dimensions.

This situation is illustrated in Fig. (2), which shows both the Standard Model and Higgs- $\phi$ production events as a function of the invariant mass of the two photons, assuming a Higgs mass of $120 \mathrm{GeV}$. The signal for Higgs- $\phi$ emission is clearly visible 

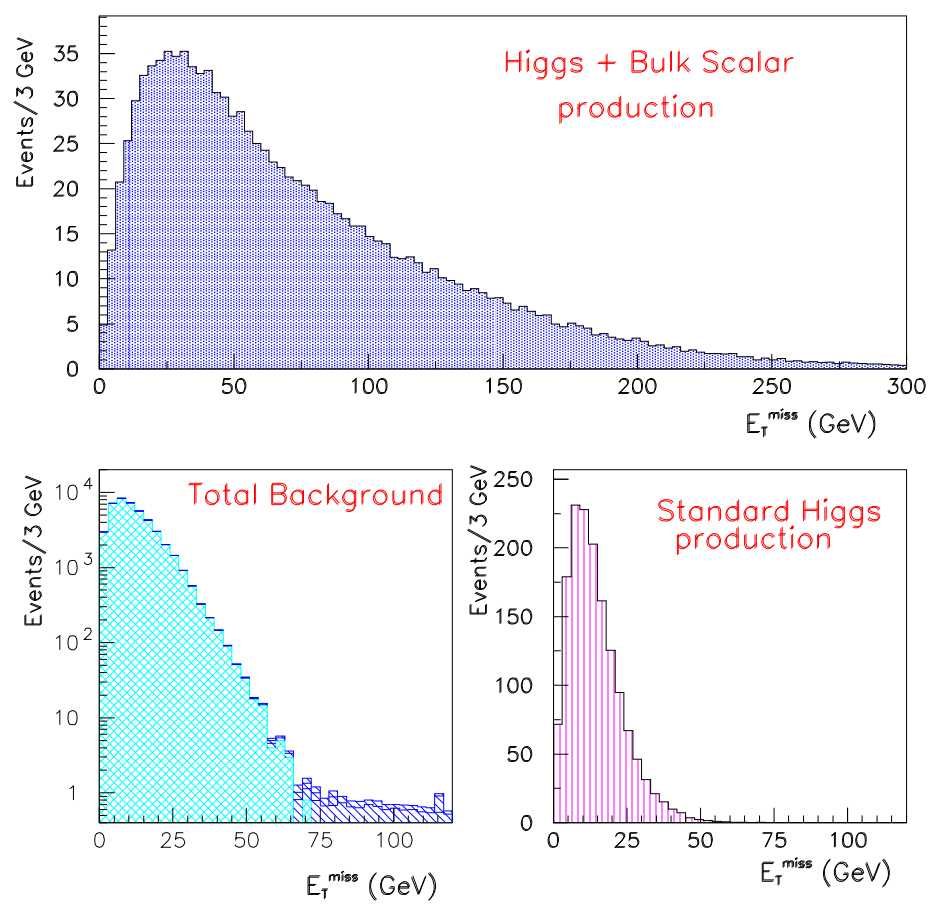

Figure 3: Distribution of $\mathbb{E}_{T}$ for the Higgs + bulk scalar signal, assuming $a=0.5$ (top), the total background (bottom-left) and the $p p \rightarrow h$ process, with $m_{H}=120 \mathrm{GeV}$ (bottomright). The plots are normalized for an integrated luminosity of $100 \mathrm{fb}^{-1}$.

on top of the irreducible background plus the standard Higgs signal. These figures are also qualitatively the same as those obtained for the standard $h \rightarrow \gamma \gamma$ process alone, which are published in the ATLAS Detector and Physics Performance Report 222.

As might be expected, and as we shall now see explicitly, those events where Higgses are produced in association with bulk scalars can be more efficiently identified by imposing a cut on the total missing energy of the event. This is shown in Fig. (3), which plots the number of background, standard Higgs and $h \phi$ events as a function of the total missing energy, $\mathbb{E}_{T}$. As this figure shows, very few background or standard Higgs events have more than $50 \mathrm{GeV}$ of missing energy, while about half of the $h \phi$ events do. The high energy tail in the background $\mathbb{E}_{T}$ distribution is due principally to the processes $Z h$ and $W h$.

The larger the missing energy required in the event, the more the background and standard Higgs events are excluded from the event sample, but also the fewer $h \phi$ events there are. Fig. (幽) shows how this trade-off scales with the effective coupling $a$, by showing the $5 \sigma$ coupling reach which is obtained as a function of the size of the missing energy cut. In this figure the standard Higgs production is counted as part of the background when computing the significance, since our goal is to identify the $5 \sigma$ discovery potential for the particular process of Higgses produced in association with $\phi$ 's. If we define a discovery signal as a sample of at least 10 events which 


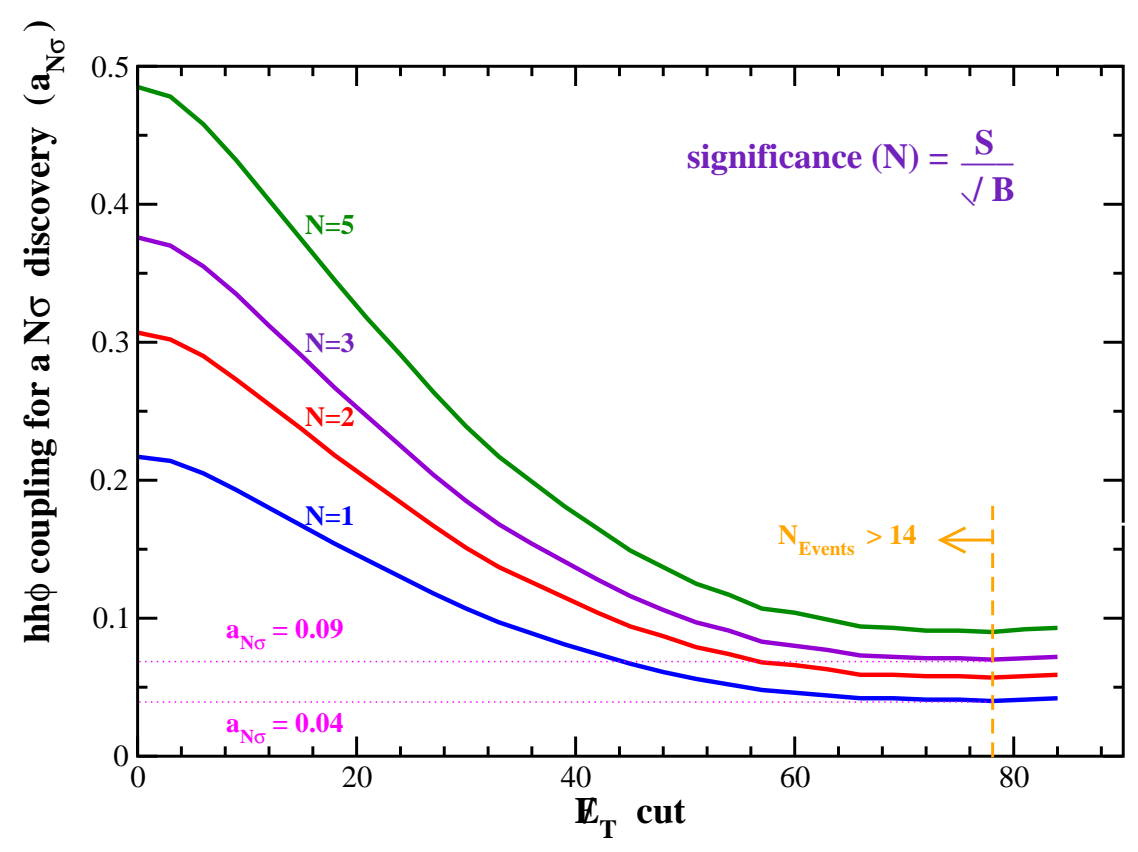

Figure 4: Value of the $h h \phi$ coupling needed for different significances of the signal, as function of a cut on $\mathbb{E}_{T}$.

has significance greater than $5 \sigma$, then the smallest coupling for which discovery is possible (with $100 \mathrm{fb}^{-1}$ of data) is $a=0.09$.

These considerations lead to the optimal missing-energy cut:

Cut 4: The missing transverse energy of the entire event must satisfy: $\mathbb{E}_{T}>78 \mathrm{GeV}$.

Imposing such a cut, 14.3 signal events are left on a total background of 8.2 events consisting of $\sim 0$ events of $\gamma \gamma+\mathrm{QCD}, 8.0$ events of $h, Z h, W h, t \bar{t} h$ and 0.2 events of $Z \gamma \gamma$ and $W \gamma \gamma$. Note that systematic errors on the measurement of the $\mathbb{E}_{T}$ may be large. A proper evaluation of this uncertainty is beyond the scope of this study but we do not expect that it will affect significantly the main conclusions. Fig. (5) shows the number of events vs invariant two-photon mass for the limiting case where $a=0.09$. We see from this figure that even this marginal case yields a clear peak at the Higgs mass, leaving unambiguous evidence for Higgs production in association with missing energy.

More generally, for larger values of $a$ than the above limit, the significance for discovery of the Higgs boson itself can be much improved, since the Standard Model backgrounds are considerably reduced. This can be seen in Fig. (6), which plots the significance of the $\gamma \gamma$ signal as a function of Higgs mass, for several choices of missing energy cut. As is clear from this figure, the curves with a nontrivial 


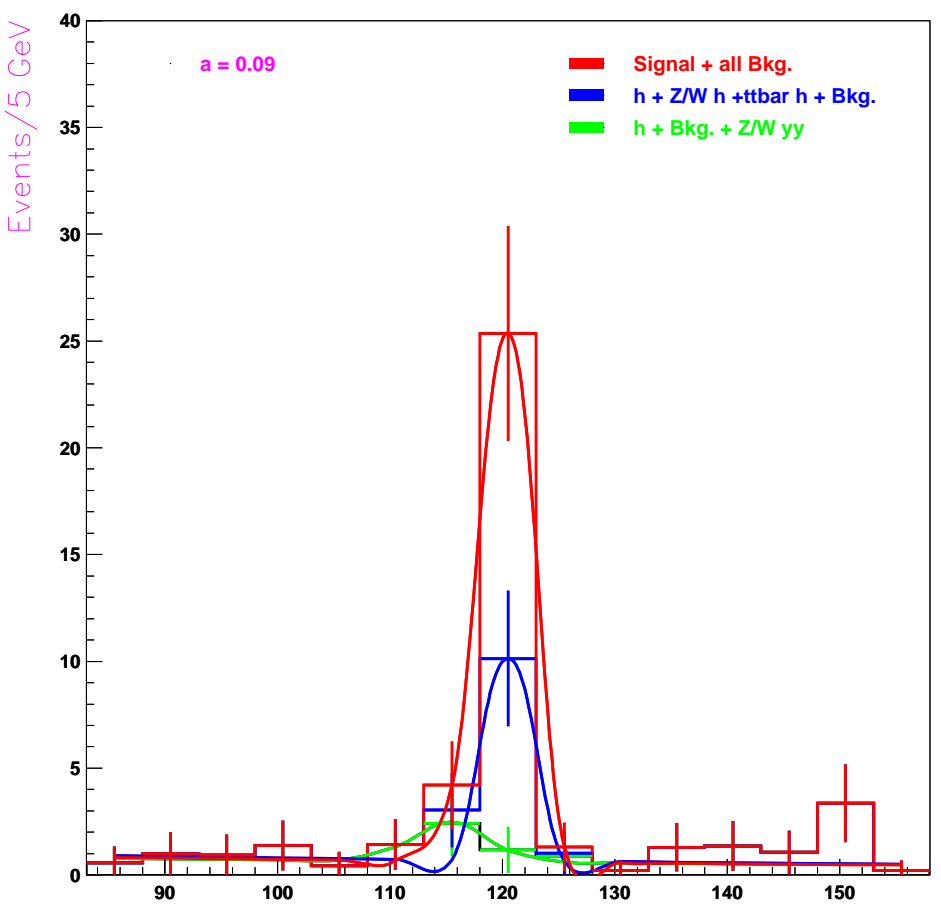

Figure 5: Number of events (including backgrounds) for $h \phi$ and standard $h$ production, as a function of the two-photon invariant mass. This plot assumes the smallest-detectable coupling $a=0.09$, and uses the optimal missing-energy cut, $\mathrm{E}_{T}>78 \mathrm{GeV}$.

missing energy cut are more significant than the one with no cut, simply because of the dramatic reduction of background relative to signal which the cut allows. The range of Higgs masses which are accessible similarly increases, as can also be seen in Fig. (6), by cutting on $\mathbb{E}_{T}$. For instance, while the mass range accessible with no cut is $105 \mathrm{GeV}<m_{h}<145 \mathrm{GeV}$, this is extended to $60 \mathrm{GeV}<m_{h}<180 \mathrm{GeV}$ or more once cuts are applied. This figure assumes $a=0.5$, but other values of the coupling are easily incorporated using the result that the missing-energy cross section scales as $a^{2}$.

We note in passing that the existence of a perturbative anomalous coupling, eq. (2.1) should not invalidate the earlier LEP searches for a Standard Model Higgs. On the other hand, this coupling should enhance the number of events found when searching with the Standard Model channel $e^{+} e^{-} \rightarrow h Z \rightarrow b \bar{b} \nu \bar{\nu}$, if a loose cut on the missing mass is applied.

\section{Conclusions}

The analysis presented here reconsiders some of the observational consequences of the existence of bulk scalars within a 6 dimensional scenario involving large extra dimensions. We have done so motivated by the recent proposals of supersymme- 


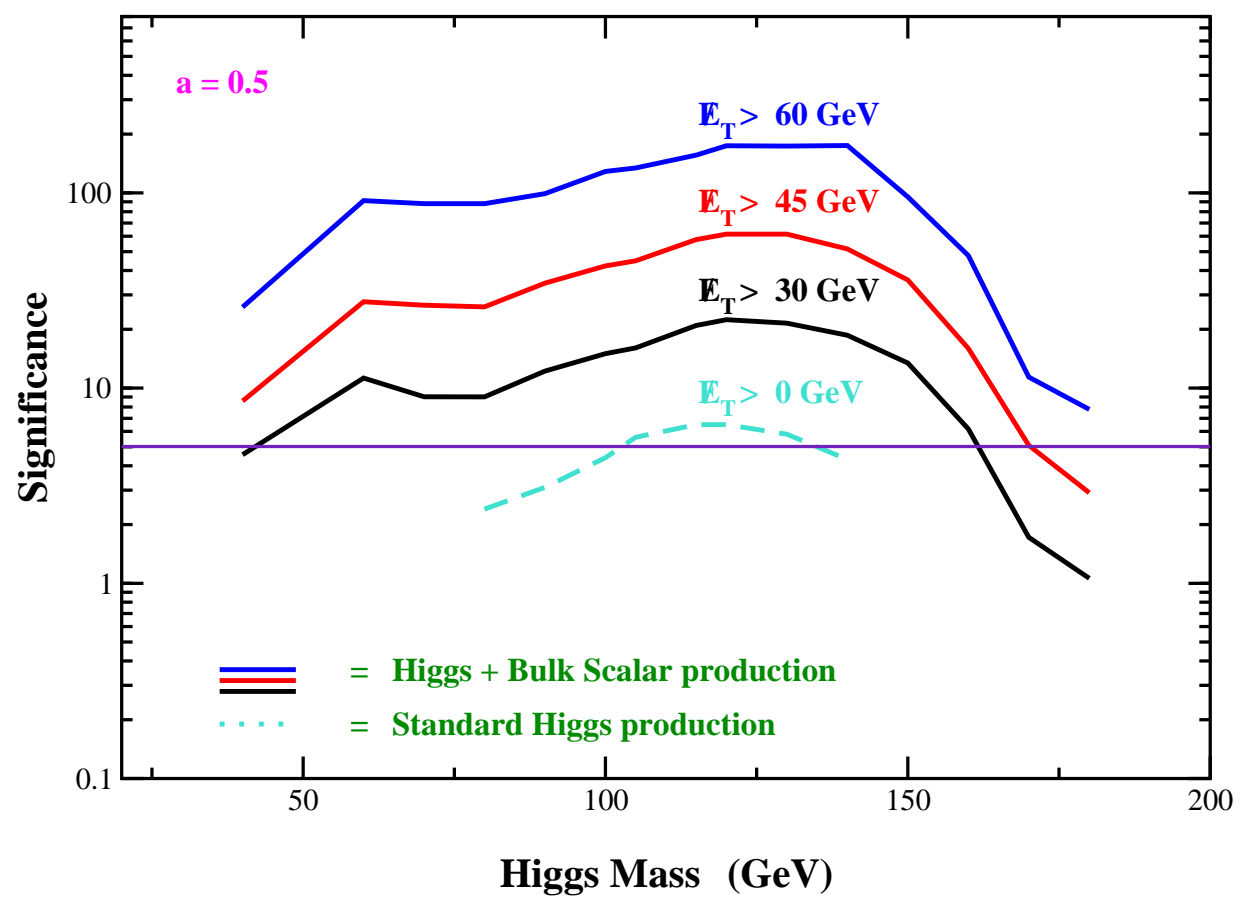

Figure 6: The significance of the $\gamma \gamma$ signal alone as function of the Higgs mass for different values of the cut on $\mathbb{E}_{T}$. The figure assumes the choices $a=0.5$ and an integrated luminosity of $100 \mathrm{fb}^{-1}$. The dotted line corresponds to what can be obtained from the standard $h \rightarrow \gamma \gamma$ process making no cut on $\mathbb{E}_{T}$. This shows that a considerable gain in Higgs reach is possible should Higgs production be possible in association with missing energy.

tric large extra dimensions, both as contributions towards understanding the small size of the cosmological constant [11] and as alternative realizations of low-energy supersymmetry [13].

We find that the lowest-dimension interaction which such a bulk scalar can have with Standard Model fields on our brane has a dimensionless coupling, $a$, and so can typically be expected to be generated with a size which is not suppressed by inverse powers of the $6 \mathrm{D}$ gravitational scale, $M_{g} \sim 10 \mathrm{TeV}$. A representative size for such a coupling as generated by one loop in 6 dimensions might then be set by loop-counting factors like $a \sim N /(4 \pi)^{3} \sim 0.01-1$, given $N \sim 10-1000$ fields circulating in the loop (which are plausible numbers for supergravity theories in 6 dimensions). The effective coupling we find resembles in some ways the effective brane-bulk mixing which is possible for the bulk metric through an effective coupling of the Higgs scalar

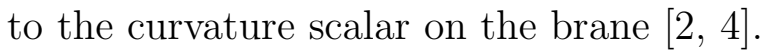

Using this effective interaction we compute the rate for the process $p p \rightarrow h \phi \rightarrow$ $\gamma \gamma \mathbb{E}_{T}$, in order to see how large an effective coupling can be detected given reasonable assumptions as to the performance of a detector like ATLAS at the LHC. Our calculation assumes that the proton reaction is dominated by the contribution of 
gluon fusion at the parton level. Given the sensitivity to $a$ which we obtain, we believe there is sufficient motivation to go back and perform more detailed studies of bulk-scalar production at colliders. It must be noted that the coupling of a bulk scalar to a more massive Higgs can lead to clean signatures, such as in the case $h \rightarrow Z Z^{(*)} \rightarrow 4 \ell$.

We numerically integrate over the appropriate parton distributions and include detector effects using existing ATLAS software. By comparing the number of signal events to the expected Standard Model backgrounds, we calculate the size of the effective couplings to which experiments at the LHC can expect to be sensitive. We find that couplings of order $a=0.5$ imply that as many Higgs particles are being produced in association with bulk scalars as are being produced without them. We find that the imposition of a missing energy cut $E_{T}>78 \mathrm{GeV}$, greatly improves the signal relative to background, and allows a $5 \sigma$ detection of the effective interaction provided the effective coupling is $a>0.09$. These limits would begin to probe the upper limit of the size of coupling which is obtained from a generic 1-loop estimate.

We also notice that the existence of Higgs production in association with missing energy is of considerable practical interest in the detection of the Higgs itself. It allows experiments to be sensitive to a much wider range of Higgs masses (at a given level of significance) than would otherwise be possible in the SM $\gamma \gamma$ decay channel.

We regard these results to be encouraging and - together with the strong motivation for bulk supersymmetry - to further motivate the study of the phenomenology of extra dimensional fields (besides the higher-dimensional metric) within the framework of large extra dimensions.

\section{Acknowledgments}

This work has been performed within the ATLAS collaboration. We have made use of physics analysis and simulation tools which are the result of collaboration-wide efforts. We thank Joaquim Matias for helpful discussions. We also want to thank A. Parker and G. Unal for useful comments. We would like to acknowledge partial funding from NSERC (Canada). C.B.'s research is also partially funded by FCAR (Québec) and McGill University.

\section{References}

[1] N. Arkani-Hamed, S. Dimopoulos and G. Dvali, Phys. Lett. B429 (1998) 263 hep-ph/9803315; Phys. Rev. D59 (1999) 086004 [hep-ph/9807344]; I. Antoniadis, N. Arkani-Hamed, S. Dimopoulos and G. R. Dvali, Phys. Lett. B 436 (1998) 257 [hep-ph/9804398].

[2] G.F. Giudice, R. Rattazzi and J.D. Wells, Nucl. Phys. B544 (1999) 3-38, [hep-ph/9811291]. 
[3] E.A. Mirabelli, M. Perelstein and M.E. Peskin, Phys. Rev. Lett. 82 (1999) 2236-2239, [hep-ph/9811337]; J.L. Hewett, Phys. Rev. Lett. 82 (1999) 4765-4768, [hep-ph/9811356]; T Han, J.D. Lykken and R.-J. Zhang, Phys. Rev. D59 (1999) 105006, [hep-ph/9811350]; I. Antoniadis, K. Benakli and M. Quiros, Phys. Lett. B460 (1999) 176-183, [hep-ph/9905311]; S. Cullen, M. Perelstein and M.E. Peskin, Phys. Rev. D62 (2000) 055012, [hep-ph/0001166].

[4] M. Battaglia, D. Dominici, J.F. Gunion and J.D. Wells, Proceedings of the Les Houches Workshop 2003: "Physics at TeV Colliders", ed. F. Boudjema (hep-ph/0402062);

A. Datta, K. Huitu, J. Laamanen and B. Mukhopadhyaya, hep-ph/0404056

[5] S. Dimopoulos and G. Landsberg, Phys. Rev. Lett. 87 (2001) 161602, [hep-ph/0106295]; S.B. Giddings and S. Thomas, Phys. Rev. D65 (2002) 056010, [hep-ph/0106219]; D.M. Eardley and S.B. Giddings, Phys. Rev. D66 (2002) 044011, [gr-qc/0201034]; R. Emparan, Phys. Lett. B526 (2002) 393-398, [hep-ph/0108060]; G.F. Giudice, R. Rattazzi and J.D. Wells, Nucl. Phys. B630 (2002) 293-325, [hep-ph/0112161]; J.L. Feng and A.D. Shapere, Phys. Rev. Lett. 88 (2002) 021303, [hep-ph/0109106]; R. Emparan, M. Masip, R. Rattazzi, Phys. Rev. D65 (2002) 064023, [hep-ph/0109287]; S. Hossenfelder, S. Hofmann, M. Bleicher and H. Stoecker, Phys. Rev. D66 (2002) 101502, [hep-ph/0109085]; Phys. Lett. B548 (2002) 73-76, [hep-ph/0112186]; K. Cheung, Phys. Rev. Lett. 88 (2002) 221602, [ hep-ph/0110163]; R. Casadio and B. Harms, Int. J. Mod. Phys. A17 (2002) 4635-4646, [hep-th/0110255]; L.A. Anchordoqui, J.L. Feng, H. Goldberg and A.D. Shapere, Phys. Rev. D65 (2002) 124027, [hep-ph/0112247]; A. Ringwald and H. Tu, Phys. Lett. B525 (2002) 135-142, [hep-ph/0111042]; M.B. Voloshin, Phys. Lett. B518 (2001) 137-142, [hep-ph/0107119]; S.N. Solodukhin, Phys. Lett. B533 (2002) 153-161, [hep-ph/0201248; T.G. Rizzo, JHEP 0202 (2002) 011, [hep-ph/0201228]; E. Kohlprath and G. Veneziano, JHEP 0206 (2002) 057, [gr-qc/0203093]; S.D.H. Hsu, Phys. Lett. B555 (2003) 92-98, [hep-ph/0203154].

[6] For recent reviews with more references, see: P. Kanti, [hep-ph/0402168]; J. Hewett and M. Spiropulu, Ann.Rev.Nucl.Part.Sci. 52 (2002) 397, [hep-ph/0205106]; G.

Azuelos et al., proceedings of the Workshop 'Physics at TeV Colliders', Les Houches, 2001 [hep-ph/0204031]; B. C. Allanach et al., proceedings of the Workshop 'Physics at TeV Colliders', Les Houches, 2003 [hep-ph/0402295].

[7] D. Atwood, C.P. Burgess, E. Filotas, F. Leblond, D. London and I. Maksymyk, Physical Review D63 (2001) 025007 (14 pages) [hep-ph/0007178];

[8] G. Azuelos, P.-H. Beauchemin and C.P. Burgess, Phenomenological Constraints on Extra-Dimensional Scalars, ATLAS publication SN-ATLAS-2004-037, [hep-ph/0401125].

[9] S. Cullen and M. Perelstein, Phys. Rev. Lett. 83 (1999) 268 [hep-ph/9903422]; V. Barger, T. Han, C. Kao, R.-J. Zhang, Phys. Lett. B461 (1999) 34-42, 
[hep-ph/9905474]; C. Hanhart, D.R. Phillips, S. Reddy, M.J. Savage, Nucl. Phys. B595 (2001) 335 [nucl-th/0007016]; J.A. Pons, D.R. Phillips and S. Reddy, Phys. Lett. B509 (2001) 1-9, [astro-ph/0102063].

[10] S. Hannestad and G. Raffelt, Phys. Rev. Lett. 87 (2001) 051301, [hep-ph/0103201]; Phys. Rev. Lett. 88 (2002) 071301, [hep-ph/0110067]; Phys. Rev. D67 (2003) 125008, Erratum-ibid. D69 (2004) 029901, [hep-ph/0304029]; S. Hannestad, Phys. Rev. D64 (2001) 023515, [hep-ph/0102290].

[11] Y. Aghababaie, C.P. Burgess, S. Parameswaran and F. Quevedo, Nuclear Physics B680 (2004) 389-414, (hep-th/0304256);

C.P. Burgess, Supersymmetric Large Extra Dimensions and the Cosmological Constant: An Update, Annals of Physics (to appear) (hep-th/0402200).

[12] Y. Aghababie, C.P. Burgess, J.M. Cline, H. Firouzjahi, F. Quevedo, G. Tasinato and I. Zavala, Journal of High Energy Physics 0309 (2003) 037 (48 pages) (hep-th/0308064).

[13] C.P. Burgess, J. Matias and F. Quevedo, MSLED: A Minimal Supersymmetric Large Extra Dimensions Scenario, (hep-ph/0404135).

[14] C.P. Burgess, M. Pospelov and T. ter Veldhuis, Nucl. Phys. B619 (2001) 709-728, [hep-ph/0011335].

[15] P. Breitenlohner and A. Kabelschacht, Nucl. Phys. B148 (1979) 96; N. Marcus and J.H. Schwarz, Phys. Lett. 115B (1982) 111; P.S. Howe, G. Sierra and P. Townsend, Nucl. Phys. B221 (1983) 331; L.J. Romans, Nucl. Phys. B269 (1986) 691-711.

[16] H. Nishino and E. Sezgin, Phys. Lett. 144B (1984) 187; Nucl. Phys. B278 (1986) 353; S. Randjbar-Daemi, A. Salam, E. Sezgin and J. Strathdee, Phys. Lett. B151 (1985) 351; A. Salam and E. Sezgin, Phys. Lett. B 147 (1984) 47; A. Salam and E. Sezgin, Phys. Lett. 147B (1984) 47; Y. Aghababaie, C.P. Burgess, S. L.

Parameswaran and F. Quevedo, JHEP 0303 (2003) 032, (hep-th/0212091).

[17] S. Randjbar-Daemi, A. Salam, E. Sezgin and J. Strathdee, Phys. Lett. B151 (1985) 351; M.B. Green, J.H. Schwarz and P.C. West, Nucl. Phys. B254 (1985) 327; J. Erler, J. Math. Phys. 35 (1994) 1819 [hep-th/9304104].

[18] C.P. Burgess, S. Godfrey, H. Konig, D. London and I. Maksymyk, Phys. Rev. D49 (1994) 6115-6147 (hep-ph/9312291).

[19] J. Ellis, M.K. Gaillard and D.V. Nanopoulos, Nucl. Phys. B106 (1976) 292; A.I. Vainshtein, M.B. Voloshin, V.I. Sakharov and M. Shifman, Sov. J. Nucl. Phys. 30 (1979) 711; E. Eichten, I. Hinchliffe, K. Lane and C. Quigg, Rev. Mod. Phys 56 (1984) 579 M. Spira, A. Djouadi, D. Graudenz and P.M. Zerwas, Nucl. Phys. 453 (1995) 17. 
[20] C.P. Burgess, Q. Matias and M. Pospelov, I.J.M.P. A17 (2002) 1841-1918, (hep-ph/9912459).

[21] T. Sjöstrand et.al., Comp. Phys. Comm. 135 (2001) 238.

[22] ATLAS Collaboration, Detector and Physics Performance Technical Design Report, vol. II, CERN-LHCC-99-15

[23] H. L. Lai et al., Eur.Phys.J. C12 (2000) 375-392 (hep-ph/9903282)

[24] E. Richter-Was, D. Froidevaux, L. Poggioli, ATLFAST 2.0, a fast simulation package for ATLAS, ATLAS-PHYS-98-131 